\title{
Evolución de la prevalencia de caries en una población infantil de Móstoles entre 1988 y 1997
}

\author{
Tapias-Ledesma MA*, Jiménez-García R**, Carrasco-Garrido P***, \\ Lamas $\mathrm{F}^{* * * * *}$, Gil de Miguel $\mathrm{A}^{* * * * *}$
}

\section{RESUMEN}

El objetivo del estudio es conocer la prevalencia de caries en una población infantil de Móstoles y los factores asociados. Hemos realizado un estudio descriptivo transversal en 363 escolares de 6 años de edad, en los colegios de la zona de influencia del Centro de Salud San Fernando (Móstoles).

Se realizó una revisión odontológica en 1997 según los criterios de la Organización Mundial de la Salud. Se recogieron variables de estado dental, sociodemográficas y de frecuencia de cepillado. Se compararon los resultados con los obtenidos en la revisión del año 1988 en esos mismos centros escolares.

Resultados. La prevalencia de caries en dentición temporal es del 24,6\% y en dentición permanente del 1,1\%. En el análisis univariante los escolares revisados en el año 1988 tienen un OR de sufrir caries de 3,48 respecto a los revisados en 1997 y los escolares de clase social baja tienen OR de 1,61 (IC95\% 1,19-2,17) respecto a los de clase media-alta.

Conclusión. La prevalencia de caries ha disminuido en la población infantil de Móstoles entre 1988 y 1997. El declinar de la caries se asocia a un mejor nivel socioeconómico. La frecuencia de cepillado ha aumentado en este periodo pero en nuestro estudio no se asocia con la disminución de la prevalencia de caries.

Palabras clave: Caries, programa preventivo, clase social, cepillado, índices de caries.

\section{SUMMARY}

The objetive .of study to asses the prevalence of caries and related factors in the infant population of Móstoles. We conducted a crossover descriptive in 363 six years old schoolchildren in Schools included in the San Fernando Health Center influence area

We conducted a dental exploration using the World Health Organization criteria. Variables such as dental state teeth brushing habits, and social-demographic aspects were collected. Results for year 1997 were compared to those obtained in the same schools in year 1988.

The prevalence of caries in 1997 for deciduous dentition is $24.6 \%$ and for permanent dentition $1.1 \%$. The non adjusted analysis shows that the schoolchildren revised in 1988 have an OR for suffering caries of 3.48 compared to those studied in 1997. Low social class is also a risk factor for caries OR=1.61(IC95\% 1.19-2.17)with respect to median-high class.

In conclusion,. the prevalence of caries in the infant population of Mostoles has decreased between 1988 y 1997. This reduction is related to an improvement in the social class of the scholars. The frequency of teeth brushing has also improved during this period but is not associated to the decrease in the prevalence of caries in this work.

Key words: Caries, preventive program, social class, teeth brushing, caries index.

Aceptado para publicación: Febrero 2005 
* $\quad$ Dr. Médico estomatólogo. Centro de Salud San Fernando (Móstoles).

** Prof. Titular Unidad de Epidemiología y Salud Pública. Facultad de Ciencias de la salud. Universidad Rey Juan Carlos.

*** Prof. Asociado. Unidad de Epidemiología y Salud Pública. Facultad de Ciencias de la salud. Universidad Rey Juan Carlos.

**** Odontólogo. Centro de Salud San Fernando (Móstoles).

***** Catedrático. Unidad de Epidemiología y Salud Pública. Facultad de Ciencias de la salud. Universidad Rey Juan Carlos.

Tapias-Ledesma MA, Jiménez-García R, Carrasco-Garrido P, Lamas F, Gil de Miguel A. Evolución de la prevalencia de caries en una población infantil de Móstoles entre 1988 y 1997. Av. Odontoestomatol 2005; 21-6:333-339.

\section{INTRODUCCIÓN}

En el año 1987 el Ministerio de Sanidad y Consumo (1) inició un programa de salud bucodental que establecía medidas (revisión odontológica, educación sanitaria, flúor tópico y selladores de fisuras), prestado desde los centros de salud y con una cobertura a la población de 6-14 años. El programa se implantó en el año 1988 en Móstoles de una manera incremental a partir de los 6 años y cada año sucesivo se añadía a los niños que cumplían 6 años (2).

Para evaluar el programa y comprobar la efectividad del mismo, iniciamos un estudio en 1988 y los resultados obtenidos fueron que más de la mitad de los escolares antes de comenzar el programa tenían caries en su dentición temporal y la frecuencia de cepillado era escasa (3).

Numerosos estudios obtienen disminución de la prevalencia de caries en los últimos años y posiblemente sea debido a la introducción de las pastas dentífricas fluoradas, a los programas de salud bucodental, aplicación de selladores oclusales y al aumento del nivel socioeconómico de la población (4-10).

El objetivo del estudio es conocer el estado de salud bucodental y factores asociados de los escolares de 6 años de Móstoles antes de comenzar el programa preventivo y comparar los resultados con los obtenidos en el año 1988.

\section{POBLACIÓN Y MÉTODOS}

Se ha realizado un estudio epidemiológico descriptivo transversal sobre 396 escolares de Móstoles de 6 años de edad. Se ofertó entrar en el estudio a los padres de todos los niños de 6 años de los once colegios que pertenece a la zona de influencia del Centro de Salud San Fernando. Los criterios de inclusión son consentimiento informado de los padres, edad de 6 años y realizarse la exploración odontológica.

Los niños fueron incluidos y revisados entre octubrediciembre del año 1997. Para determinar el estado dental hemos realizado una exploración odontológica siguiendo los criterios de la Organización Mundial de la Salud (OMS) (12), con espejo plano, sonda de punta fina y luz natural. Rellenamos una ficha individual donde anotamos en un odontograma los diferentes estados dentales tanto para la dentición temporal como permanente.

El diagnóstico para el estado dental se hizo siguiendo los criterios y códigos de la OMS (13), que considera diente sano a aquel que no presenta evidencia de caries actual ni de caries tratada, todo hallazgo sugerente de que puede evolucionar a caries es considerado como diente sano. Definimos caries dental como aquella lesión de un diente que presenta el esmalte socavado con suelo y paredes cavitarias reblandecidas. Las obturaciones con material temporal se consideran caries dental.

Un mismo explorador (médico estomatólogo) ha revisado a todos los escolares.

Para medir y comparar los estados dentales hemos utilizado la prevalencia de caries en dentición temporal y permanente, así como, los índices de caries en dentición temporal (cod) y permanente (CAOD).

Como variables sociodemográficas vamos a medir el 
sexo y la clase social, en esta última hemos utilizado la clasificación abreviada de la Sociedad Española de Epidemiología, atendiendo a la profesión del cabeza de familia (14).

La frecuencia de cepillado se ha recogido como dicotómica según se cepillen los dientes diariamente o no.

Vamos a medir los diferentes estados dentales y factores asociados de los escolares que cumplan los criterios de inclusión en 1997 y los compararemos con los resultados obtenidos en la revisión del año 1988.

También hemos unificado las bases de datos de las revisiones del 1988 y la de 1997 para realizar un análisis estadístico univariante y multivariante de las variables asociadas a la presencia de caries en los niños a la edad de seis años. Las variables estudiadas en esta fase son: el año de la revisión, el sexo, la clase social (dicotomizada en media-alta y baja) y los hábitos de cepillado. Como medidas de asociación utilizaremos el odds ratio (OR) y sus intervalos de confianza al 95\% (IC95\%). Para el análisis multivariate emplearemos regresión logística. Para realizar los cálculos estadísticos y epidemiológicos utilizaremos el programa SPSS V. 10.0.

\section{RESULTADOS}

De los 396 escolares que entraron en el estudio, fueron excluidos 43 , por lo que la población estudiada quedó constituida por 353 escolares de los cuales 162 son niñas y 191 niños.

Al describir la población a la edad de 6 años (tabla 1) y al comparar a los escolares según año de revisión, la prevalencia de caries en el año 1997 es del $24,6 \%$ y en el año 1988 era del 53,9\%. Encontramos diferencias estadísticamente significativas en la prevalencia de caries en dentición temporal y permanente. También existen diferencias en la clase social y frecuencia de cepillado. El índice cod es de 1,95 en el año 1988 y en el año 1997 obtenemos un índice cod de 0,69, existen diferencias estadísticamente significativas. En el índice CAOD también encontramos diferencias estadísticamente significativas (tabla 2).

En el análisis univariante (tabla 3) obtenemos un OR del 3,57 (IC95\% 2,65-4,81) de presentar caries para los escolares revisados en el año 1988 respecto a los de 1997. No se obtienen diferencias por sexo. En cambio los escolares que no se cepillan diariamente tienen un riesgo de 1,59 veces mayor de tener caries

\section{TABLA 1.- DESCRIPCIÓN DE LOS ESCOLARES REVISADOS EN LOS AÑOS 1988 Y 1997 A LA EDAD DE 6 AÑOS. COMPARACIÓN DE LAS DIFERENTES VARIABLES}

\begin{tabular}{|c|c|c|c|c|c|c|}
\hline & \multirow[b]{2}{*}{ Categoría } & \multicolumn{2}{|c|}{$\begin{array}{l}\text { Escolares revisados en } 1988 \\
n=512\end{array}$} & \multicolumn{2}{|c|}{$\begin{array}{l}\text { Escolares revisados en } 1997 \\
n=353\end{array}$} & \multirow[t]{2}{*}{$\mathbf{P}$} \\
\hline & & Frecuencia & $\%$ & Frecuencia & $\%$ & \\
\hline Sexo & $\begin{array}{l}\text { Niño } \\
\text { Niña }\end{array}$ & $\begin{array}{l}254 \\
258\end{array}$ & $\begin{array}{l}49,6 \\
50,4\end{array}$ & $\begin{array}{l}162 \\
191\end{array}$ & $\begin{array}{l}45,6 \\
54,14\end{array}$ & n.s. \\
\hline Clase social & $\begin{array}{c}\text { Alta } \\
\text { Media } \\
\text { Baja }\end{array}$ & $\begin{array}{r}20 \\
115 \\
377 \\
\end{array}$ & $\begin{array}{r}3,9 \\
22,5 \\
73,6 \\
\end{array}$ & $\begin{array}{r}17 \\
123 \\
213 \\
\end{array}$ & $\begin{array}{r}4,8 \\
34,8 \\
60,3 \\
\end{array}$ & $<0,001$ \\
\hline Caries DT & $\begin{array}{l}\text { Sí } \\
\text { No }\end{array}$ & $\begin{array}{l}276 \\
236\end{array}$ & $\begin{array}{l}53,9 \\
46,1\end{array}$ & $\begin{array}{r}87 \\
266\end{array}$ & $\begin{array}{l}24,6 \\
75,4\end{array}$ & $<0,001$ \\
\hline Caries DP & $\begin{array}{l}\text { Sí } \\
\text { No }\end{array}$ & $\begin{array}{r}37 \\
475\end{array}$ & $\begin{array}{r}7,2 \\
92,8\end{array}$ & $\begin{array}{r}4 \\
3,49\end{array}$ & $\begin{array}{r}1,1 \\
98,9\end{array}$ & $<0,001$ \\
\hline Capillado diario & $\begin{array}{l}\text { Sí } \\
\text { No }\end{array}$ & $\begin{array}{r}93 \\
419\end{array}$ & $\begin{array}{l}18,2 \\
81,8\end{array}$ & $\begin{array}{l}186 \\
167\end{array}$ & $\begin{array}{l}52,7 \\
47,3\end{array}$ & $<0,001$ \\
\hline
\end{tabular}

DT: dentición temporal. DP: dentición permanente. P: significación estadística. 


\begin{tabular}{|c|c|c|c|c|c|}
\hline \multicolumn{6}{|c|}{$\begin{array}{c}\text { TABLA 2.- DESCRIPCIÓN DE LOS ESCOLARES } \\
\text { REVISADOS EN LOS AÑOS } 1988 \text { Y } 1997 \text { A LA } \\
\text { EDAD DE } 6 \text { AÑOS. COMPARACIÓN DE LAS } \\
\text { DIFERENTES VARIABLES }\end{array}$} \\
\hline & \multicolumn{2}{|c|}{$\begin{array}{l}\text { Revisión } \\
\text { año } 1988\end{array}$} & \multicolumn{2}{|c|}{$\begin{array}{c}\text { Revisión } \\
\text { año } 1997\end{array}$} & \multirow[t]{2}{*}{$\mathbf{P}$} \\
\hline & Media & sd & Media & sd & \\
\hline Cod & 1,95 & 2,56 & 0,69 & 1,54 & $<0,001$ \\
\hline CAOD & 0,12 & 0,52 & 0,01 & 0,19 & $<0,001$ \\
\hline
\end{tabular}

Sd: desviación estándar

y la clase social baja presenta un OR de 1,61 (IC95\% 1,19-2,17).

En el análisis multivariante se obtiene un OR de presentar caries de 3.48 (IC95\% 2,53-4,80) para los escolares incluidos el año 1988 respecto a los reclutados en 1997, y un OR de 1,39 (IC95\% 1,02-1,90) para la clase social baja respecto a la media-alta. Sin embargo, no se obtiene significación para la frecuencia de cepillado.

\section{DISCUSIÓN}

La mayoría de las pérdidas (43) fueron debidas a falta de consentimiento informado y no creemos puedan sesgar nuestros resultados. El número de escolares del año 1988 es mayor que el del año 1997, lo que demuestra que la natalidad en Móstoles ha disminuido, ya que los escolares fueron revisados en los mismos once colegios que pertenece a la zona de influencia del Centro de Salud San Fernando.

La prevalencia de caries en dentición temporal en el año 1997 es del 24,6\% menor que la obtenida en el año 1988 que fue del 53,9\%, existiendo diferencias estadísticamente significativas lo que demuestra que la salud bucodental ha mejorado en estos últimos años y posiblemente es debido en parte a la influencia del programa de preventivo de salud bucodental $(1,2)$.

La prevalencia en dentición permanente del año 1997 es menor que la del año 1988, existiendo diferencias estadísticamente significativas, esto puede ser debido a la disminución de caries en dentición temporal, ya que tener caries en dientes deciduos es un factor de riesgo de padecer caries en la dentición permanente $(4,15)$.

Respecto a la clase social se observa una mejoría en la misma siendo en el año 1997 donde se registra un aumento de la clase social media y disminuyendo la clase social baja, lo que indica que el aumento del nivel socioeconómico de la población de Móstoles ubicada en la zona de influencia de nuestro Centro de Salud (tabla 1).

Un 52,7\% de los escolares en el año 1997 se cepillan diariamente, mientras que en el año 1988 sólo se cepillaban diariamente el $18,2 \%$, esto demuestra que la frecuencia de cepillado ha mejorado y posiblemente sea debido a la influencia del programa de salud bucodental, ya que todos los años damos

\begin{tabular}{|l|c|c|c|c|c|}
\hline \multicolumn{5}{|c|}{ TABLA 3.- ANÁLISIS UNIVARIANTE Y MULTIVARIANTE SEGÚN CARIES EN DENTICIÓN TEMPORAL } \\
PARA LAS VARIABLES: AÑO DE ESTUDIO, SEXO, FRECUENCIA DE CEPILLADO Y CLASE SOCIAL \\
\hline & Variables & OR Crudo & IC95\% & OR Ajustado & IC95\% \\
\hline Año & 1997 & 1 & & 1 & \\
& 1988 & 3,57 & $2,65-4,81$ & 3,48 & $2,53-4,80$ \\
\hline Sexo & Niño & 1 & & 1 & \\
& Niña & 1,03 & $0,78-1,35$ & 1,07 & $0,80-1,42$ \\
\hline Cepillado diario & Sí & 1 & & 1 & $0,69-1,36$ \\
\hline Clase social & No & 1,59 & $1,18-2,14$ & 0,98 & $1,02-1,90$ \\
\hline
\end{tabular}

OR: odds ratio. IC95\% intervalos de confianza al 95\%. 
charlas de educación sanitaria a los niños y a sus padres en los colegios y en el Centro de Salud. De todas formas, hay autores que refieren que una de las causas de la disminución de caries en países industrializados ha sido el cepillado con pasta dentífrica fluorada $(4,5,16,17)$.

Al comparar la prevalencia de caries con otros estudios, encontramos un $79 \%$ en escolares de Cádiz, $66 \%$ en escolares madrileños, $53,33 \%$ en la Comunidad de Castilla y León, $45.4 \%$ en escolares de Región de Murcia (18-21).

Al comparar los índices de caries en dentición temporal, encontramos un índice cod de 2,10 en escolares asturianos, 1.84 en escolares de Madrid, 1,34 en Cataluña, 0,96 en escolares de Barcelona, 2,07 en niños de Vigo y 1.7 en escolares de Inglaterra y Gales $(7,19,22-25)$.

En el análisis univariante se obtiene un riesgo de 1,59 veces mayor para los niños que no se cepillan, esto confirma que el cepillado con pasta dentífrica fluorada disminuye la caries dental (4). Sin embargo, al realizar el análisis multivariante no encontramos mayor riesgo entre los niños que se cepillan con frecuencia que de los que no se cepillan diariamente, esto puede ser debido a que dicho análisis se realiza para la dentición temporal y los niños suelen comenzar a cepillar los dientes a partir de los 3 años, aunque nosotros indicamos que el cepillado debe comenzar a edades mas tempranas alrededor de los 2 años, pero siempre supervisado por los padres. También puede ocurrir que al revisar a los niños le preguntamos si se cepillan o no los dientes diariamente y como suelen ir en grupos de cinco, es probable que no nos hayan dicho la verdad, es decir que sea un sesgo de información. Debemos tener en cuenta que la primera revisión la efectuamos a los 6 años y observamos que hay niños que han acudido al dentista para tratarse las caries (tienen obturaciones) y posiblemente a partir de ese momento comiencen a lavarse los dientes incluso más frecuente que los niños sin caries, esta puede ser otra causa de la pérdida de la significación estadística. Sin embargo, debemos pensar que exista algún factor de confusión que pueda influir en la variable frecuencia de cepillado como son ciertos estilos de vida (consumo de golosinas), que no hemos recogido en el estudio.
Al realizar el análisis multivariante obtenemos que los escolares del año 1988 tenían 3,48 veces mayor riesgo de padecer caries que los escolares de 1997, esto demuestra que la salud bucodental en los escolares de 6 años de Móstoles ha mejorado en los últimos nueve años. No encontramos diferencias en la variable sexo, corrobora otros estudios donde no se encuentren diferencias entre niños y niñas respecto a la dentición temporal $(2,26)$, sin embargo, hay estudios donde se obtienen diferencias según sexo, teniendo mayor prevalencia las niñas (27).

Los escolares de clase social baja en el análisis univariante tienen un 1,61 mayor riesgo de padecer caries que de los clase social alta, lo que confirma la clase social baja como un factor de riesgo de padecer caries (8). Al realizar el análisis multivariante la clase social baja presenta un riesgo de 1,39 veces mayor de presentar caries, lo que corrobora que pertenecer a la clase social baja es un factor de riesgo de padecer caries dental. Por lo tanto, el mejor nivel socioeconómico posiblemente conlleve a una disminución de la caries dental como ocurre en los países industrializados $(4,28,29)$.

En conclusión, la prevalencia de caries ha disminuido en los escolares de 6 años de Móstoles, lo que contribuye a una mejor salud bucodental. Se ha producido un aumento del nivel socioeconómico el cual influye en la disminución del riesgo de caries. Los escolares del año 1988 presentan un riesgo de caries 3,48 veces mayor que los escolares del año 1997, lo que confirma el declinar de la caries en nuestra población infantil.

\section{BIBLIOGRAFÍA}

1. Ministerio de Sanidad y Consumo. Programa de Salud Bucodental. Madrid: Dirección de Planificación Sanitaria, 1987.

2. Tapias MA, Gil A, Domínguez V. Prevalencia de caries en una población infantil de Móstoles. Av Odontoestomatol 2000; 16: 241-50.

3. Tapias MA. Incidencia de caries en una población infantil de Móstoles: estudio de cohortes prospectivas [tesis doctoral]. Madrid: Universidad Complutense de Madrid. Facultad de Medicina, 1999. 
4. Rioboo R. La evolución de la salud bucodental en la población europea durante la última década: variables implicadas. Av Odontoestomatol 1988; 199-34.

5. Horowitz AM. The publics oral health: the gaps between why we know and what we practice. Adv Dent Res 1995; 9: 91-5.

6. Tapias Ledesma MA, Jimenez-Garcia R, Lamas F, Gil de Miguel A. Efectividad de los selladores de fisuras en una población infantil con alto riesgo de presentar caries. Aten Primaria 2002; 30: 150-6.

7. Dolado I, Casañas P, Nebot M, Manau C. Prevalencia de caries y factores asociados en escolares de 12 años de Barcelona. Aten Primaria 1996; 18: 25-33.

8. Freire MCM, Melo RB. Dental caries prevalence in relation to socio economic status of nursery school children in Goiania-go, Brazil. Community dent oral epidemiol 1996; 24: 357-61.

9. Kumar JV, Davila ME, Green EL, Lininger LL. Evaluation of a school-based sealant program in New York State. J Public Health Manag Pract 1997; 3: 43-51.

10. Ismail AI, Gagnon P. A longitudinal evaluation of fissure sealants applied in dental practices. J Dent Res 1995; 74: 1583-90.

11. Hausen H, Karkkainen S, Seppa L. Application of the high-risk strategy to control dental caries. Community Dent Oral Epidemiol 2000; 28: 2634.

12. World Health Organization. Oral Health Surveys: Basic Methods. 3rd ed. Geneva:WHO 1987.

13. World Health Organization. Oral Health Surveys: Basic Methods. 4th ed. Geneva:WHO 1997.

14. Alvarez A, Alonso J, Domingo A, Regidor E. La medición de la clase social en ciencias de salud. Informe de un grupo de trabajo de la Sociedad Española de Epidemiología. Colección de informes técnicos serie Y 1995; 106-11.

15. Scheutz F, Frydenberg M, Matee MI, Poulsen S. The effect of choosing different units of analysis when estimating risk of presence of dental caries in the primary dentition. Community Dent Health 2003; 20: 27-33.

16. Beltran ED, Burt BA. Efectos pre y posteruptivos del fluor en la disminución de la caries. Arch Odontoestomatol Preven Comun 1989; 1: 36-45.

17. Manau C. Epidemiologia oral y odontología preventiva: revisión de la literatura 1989. Arch Odontoestomatol Preven Comun 1990; 4: 92 106.

18. Romero MM, Serrano A, Alvarez-Osorio M, Zafra JA. Prevalencia de la caries dental en la población de 6 años de San Fernando (Cádiz). Aten Primaria 1996; 18: 431-5.

19. Rioboo R. Estudio epidemiologico de las enfermedades bucodentarias en una zona de la provincia de Madrid (zona no fluorada). Rev San Higiene Publ 1980; 54: 1043-60.

20. Anitua MA. Estudio epidemiologico de la salud bucodental de los escolares de Castilla y León. Junta de Castilla y León. Consejería de Sanidad y Bienestar Social. Vallodolid. 1995.

21. Navarro JA. Encuesta de salud bucodental en escolares de la Región de Murcia. Región de Murcia. Consejería de Sanidad. 1990.

22. Alvarez A, Alvarez-Riesgo JA, Villa MA, Quiros JR. Caries dental en la población escolar del Principado de Asturias. Arch Odontoestomatol Preven Comun 1994; 10: 201-9.

23. Cuenca E, Batalla J, Manau C, Taberner JL, Salleras L. Encuesta de prevalencia de caries entre los escolares de Cataluña. $1^{a}$ parte. Arch Odontoestomatol Preven Comun 1992; 4: 1-6.

24. Iglesias Parada IM.La salud oral en la dentición primaria: (1). Estudio sobre la caries dental en una muestra de 441 niños de Vigo. Av Odontoestomatol 2002;18: 285-95

25. Downer MC. Time trends in caries experience of children in England and Wales. Caries Res 1992; 26: 466-72.

26. Fuentes S, Gutierrez C. Caries dental en una población escolar con programa de enjuagues 
periodicos de fluoruro sodico. Aten Primaria 1995; 16: 55-62.

27. Garcia-Camba JM, Oñorbe M. La salud bucodental de la población infantil en la Comunidad de Madrid. Dirección General de Prevención y Promoción de la Salud. Consejeria de Salud. Documento Técnico No 3. $2^{\text {a }}$ ed. 1994.

28. Brown LJ, Selwitz RH. The impact of recent changes in the epidemiology of dental caries on guidelines for the use of dental sealants. J Public Health Dentistry 1995; 55: 274-91.

29. Newbrun E. Preventing dental caries: current and prospective strategies. JADA 1992; 123: 68-73.

\section{CORRESPONDENCIA}

Dr. Miguel Angel Tapias Ledesma

Centro de Salud San Fernando

C/ Azorín, 3.

CP. 28935 Madrid

e-mail:mtapiasl@infomed.es 